\title{
AN ENERGY EFFICIENT ANALYSIS OF S-MAC AND H-MAC PROTOCOLS FOR WIRELESS SENSOR NETWORKS
}

\author{
P.T.Kalaivaani ${ }^{1}$ and A.Rajeswari ${ }^{2}$ \\ ${ }^{1}$ Department of Electronics and Communication Engineering, \\ MNM Jain Engineering College, Anna University, Chennai, India \\ ptkalaivaani@gmail.com \\ ${ }^{2}$ Department of Electronics and Communication Engineering, \\ Coimbatore Institute of Technology, Coimbatore, India \\ Rajeswari.ece.cit@gmail.com
}

\begin{abstract}
Wireless Sensor Networks (WSNs) is an interesting topic to the researchers because of its various applications. The applications are health monitoring and environmental monitoring, Industrial Process Monitoring, Target detection, Target tracking, Energy Efficiency, Disaster Management and Military Security Systems. The wireless medium requires highly optimized medium Access Protocols to avoid interferences. Limited resources have driven the research towards energy consumption of MAC functionalities. Two Medium Access Control (MAC) protocol performances are analyzed by using the MAC layer frame work for wireless sensor networks. In this paper an energy efficient analysis of S-MAC and $H$ MAC protocols for wireless sensor networks is proposed with spatial correlation concept. Two MAC protocols such as Sensor MAC (S-MAC) and Hybrid MAC (H-MAC) protocols are proposed to analyze the performance of Wireless Sensor Network using four different Parameters such as End to End delay, Packet Delivery Ratio, Packet Drop Rate and Energy Consumption. Performance analysis is carried out by using the simulation tool NS2.
\end{abstract}

\section{KEYWORDS}

Medium Access Control (MAC), Wireless Sensor Networks (WSNs), Sensor MAC (S-MAC), Hybrid MAC $(H-M A C)$,

\section{INTRODUCTION}

Today the major difficulty in the deployment phase of wireless Sensor Networks is their dependence on limited battery power. The major role of Medium Access control Protocols is to coordinate access to and transmission over a medium that is common to other nodes in Wireless Sensor Networks. The number of nodes in the entire Wireless Sensor Networks design as well as the number of neighboring nodes in WSNs play a vital part in WSN MAC design. The MAC plays a central Part in the WSN design because it controls the active and sleeping state of each node. The prevalent source of energy waste in WSNs are Idle Listening and Over hearing and transmission, Collisions, Over emitting. The properties that must be considered to design a good MAC protocols are categorized into two types. They are primary and secondary attributes. Primary attributes are energy efficiency and scalability and adaptability to changes such as node density, network size and topology. Latency, throughput and bandwidth utilization are the secondary attributes. Basically MAC layer Controls the transmission and reception of the actual 
packets between the nodes in the Network. It also controls the network interface between a host when the connection is established or the transaction is performed in a network. In this paper an Energy Efficient analysis of S-MAC and HMAC protocols for Wireless Sensor Networks is discussed.

Related work is briefly discussed in section II. In section III,IV,V,VI, The Network model and the concept of Sensor MAC, Hybrid MAC protocols, Spatial Correlation in WSNs are explained. In section VII, the results are discussed. In section VIII, conclusion and future work are given.

\section{RELATED WORK}

A brief Literature survey has been given in the following section

ERMAC is a hybrid MAC Protocol, it is developed from TDMA and CSMA approaches. ERMAC allows the node to join or leave the network [1].The survey and analysis of most energy efficient MAC protocol are given with comparison [2]. Demonstration of wireless sensor network with SMAC protocol is given. A modification in that protocol proves that some nodes to stay awake longer than the other nodes. The modified protocol improves the energy efficiency and increases the life time of wireless sensor networks [3]. The outline of Wireless Sensor Network and MAC, routing protocol is proposed and its analyzed using MATLAB [4]. Properties of sensor networks that are complex for the design of MAC layer protocol is explained. Several MAC protocols has been listed out with their with their strength and weakness[5].Efficient Hybrid MAC with an embedded cross layer optimization solution with an embedded cross layer optimization technique has been given. HMAC combines energy efficient feature of contention based and TDMA based MAC protocols that adapts a short frame structure for packet delivery [7]. Aiming to highlight the energy waste sources in Wireless Sensor Networks. SMAC and Timeout MAC and TEEM are the three different MAC protocols that are investigated in this paper [9].Simulation study of performance of several conventional and hybrid MAC protocol has been proposed. For the specific network conditions, the relative performance of each protocol has been analyzed [10].

\section{NETWORK MODEL}

An architecture of WSNs consists of radio and command center. Sensors have small batteries and they are deployed in unattended setup. Therefore replacing sensor's battery is not practical. So the energy efficient design and management of sensor network are real challenges for the researchers. The hardware elements of a WSNs are shown in Figure1.

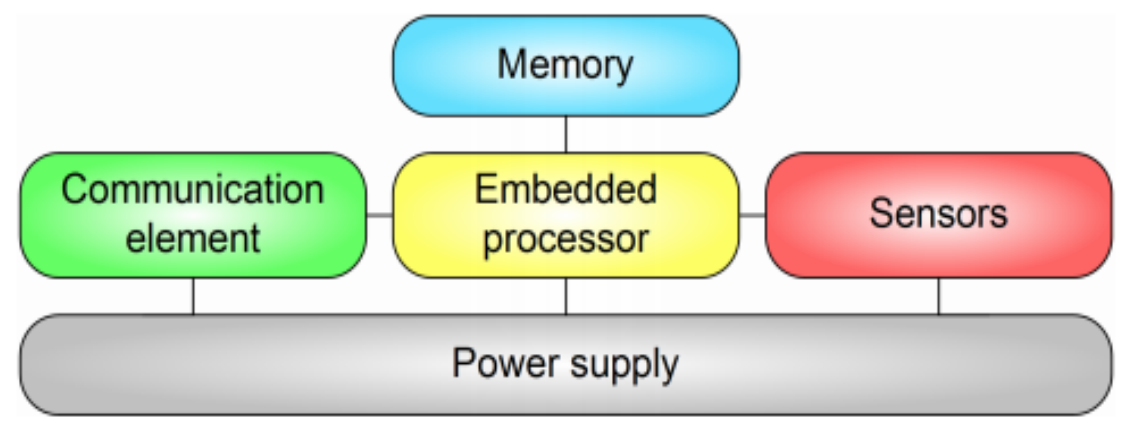

Figure 1. Hardware Elements of a Wireless sensor Networks 
Memory, Communication element, Embedded Processor, sensors and Power Supply form the hardware elements of a wireless sensor network.

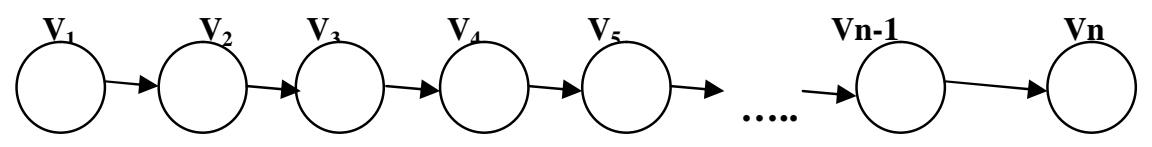

Figure 2. Wireless Sensor Network Model

The total number of nodes available in sensor field is $v_{1}$ to $v_{n}$. Each has their own residual energy. The protocol stack and the cross layer information exchange of the sensor nodes are given in Figure 3 and 4.

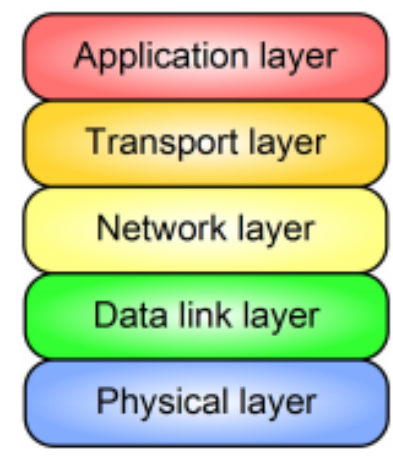

Figure 3. Protocol Stack

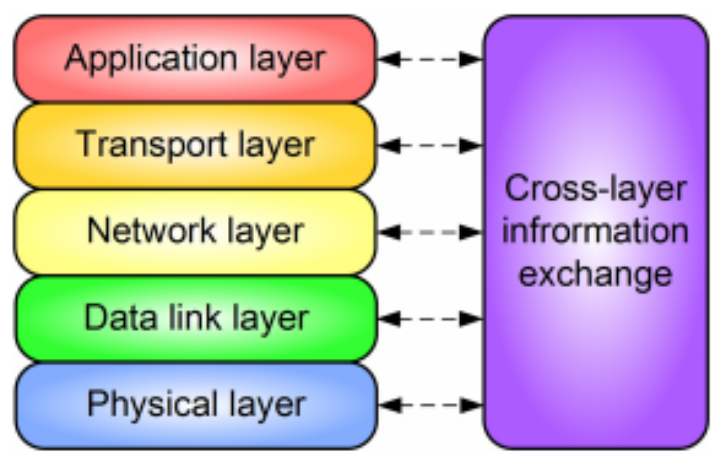

Figure 4. Cross layer information exchange

The protocol stack is shown in Figure 3. It consists of application Layer, Transport layer, Network layer, Data link layer and Physical layer. In Figure 4, the cross layer information exchange has been given. It consists of five layers which are available in protocol stack and all the layers are connected to cross layer information exchange. Two MAC protocols are proposed in this work. They are S-MAC and HMAC. The protocols are discussed in the following section.

\section{SENSOR MAC}

S-MAC is a contention based protocol proposed for energy constrained in a network. S-MAC protocol provides mechanism to get around idle listening, collisions and over hearing. It adopts a periodic wakeup scheme, i.e., each node alternates between a fixed length listen period and fixed length sleep period. S-MAC includes periodic listen and sleep and collision and over hearing avoidance and message passing. The energy waste caused by idle listening is reduced by sleep schedule in S-MAC. In S-MAC, nodes form virtual clusters with common sleep schedule, so all the clusters wake up and start communicating at the same time. The channel is divided into an active and sleep period. Potential energy saving is determined by the ratio between the active and passive periods during the active period. The node starts synchronization sequence is called synchronizer. It emits an SYNC packet which synchronizes all nodes inside the virtual cluster. Collision avoidance is achieved by the carrier sense and by the data exchange schemes RTS/CTS/DATA/ACK [6].The active and sleep period as of S-MAC protocols are shown in Figure 5 for three different number of nodes.

The entire latency over $\mathrm{N}$ hopes are given by the formula, 
International Journal of Computer Networks \& Communications (IJCNC) Vol.5, No.2, March 2013

$$
\mathrm{L}(\mathrm{N})=\sum_{k=0}^{N}\left(t_{s, n}+t_{d}\right)
$$

$\mathrm{N}$ is the number of hops, $\mathrm{t}_{\mathrm{s}, \mathrm{n}}+\mathrm{t}_{\mathrm{d}}$ backoff and transmission delay, $\mathrm{n}$ represents the current hop value Average Latency over $\mathrm{N}$ hop is given by, $\mathrm{E}[\mathrm{L}(\mathrm{N})]=\mathrm{N}\left(t_{s, n}+t_{d}\right)$

$$
D_{n}=t_{s l, n}+t_{s, n}+t_{d}
$$

Where $T>t_{d}$ and $t_{s, n}$ is the sleep delay. In S-MAC without adaptive listening contention only starts at the beginning of each frame. This is given by

$$
\mathrm{T}=\mathrm{t}_{\mathrm{s}, \mathrm{n}-1}+\mathrm{t}_{\mathrm{d}}+\mathrm{t}_{\mathrm{sl}, \mathrm{n}}
$$

So the sleep delay at hop $\mathrm{n}$ is given by

$$
t_{\mathrm{s} l, n}=T-\left(t_{s, n-1}+t_{d}\right)
$$

Substituting the equation (5) in (3)

$$
D_{n}=T+t_{s, n}-t_{s, n-1}
$$

A packet can be generated on the source at any time within a frame, so the sleep delay on the first hop tsl,1, is a random variable whose value lies in $(0, T)$.suppose tsl,1 is uniformly distributed in $(0, \mathrm{~T})$.It means value is $\mathrm{T} / 2$.Combining it with equation (6), the overall delay of a packet over $\mathrm{N}$ hop is given by,

$$
\begin{aligned}
\mathrm{D}(\mathrm{N}) & =\mathrm{D}_{1}+\sum_{n=2}^{N} D_{n} \\
& =\mathrm{t}_{\mathrm{s}, 1}+\mathrm{t}_{\mathrm{s}, 1}+\mathrm{t}_{\mathrm{d}}+\sum_{n=2}^{N}\left(T+t_{s, n}-t_{s, n-1}\right) \\
& =\mathrm{t}_{\mathrm{sl}, 1}+(\mathrm{N}-1) \mathrm{T}+\mathrm{t}_{\mathrm{s}, \mathrm{N}}+\mathrm{t}_{\mathrm{d}}
\end{aligned}
$$

The average latency of S-MAC without adaptive listen over $\mathrm{N}$ hops is given by

$$
\begin{aligned}
\mathrm{E}[\mathrm{D}(\mathrm{N})] & =\mathrm{E}\left[\mathrm{t}_{\mathrm{sl}, 1}+(\mathrm{N}-1) \mathrm{T}+\mathrm{T}_{\mathrm{s}, \mathrm{N}}+\mathrm{t}_{\mathrm{d}}\right] \\
& =\mathrm{T} / 2+(\mathrm{N}-1) \mathrm{T}+\mathrm{T}_{\mathrm{s}}+\mathrm{t}_{\mathrm{d}} \\
& =\mathrm{NT}-\mathrm{T} / 2+\mathrm{t}_{\mathrm{s}}+\mathrm{t}_{\mathrm{d}}
\end{aligned}
$$

From the equation (8) we can observe that the multihop latency increases with the number of hops in SMAC when each node strictly follows its sleep schedule. 


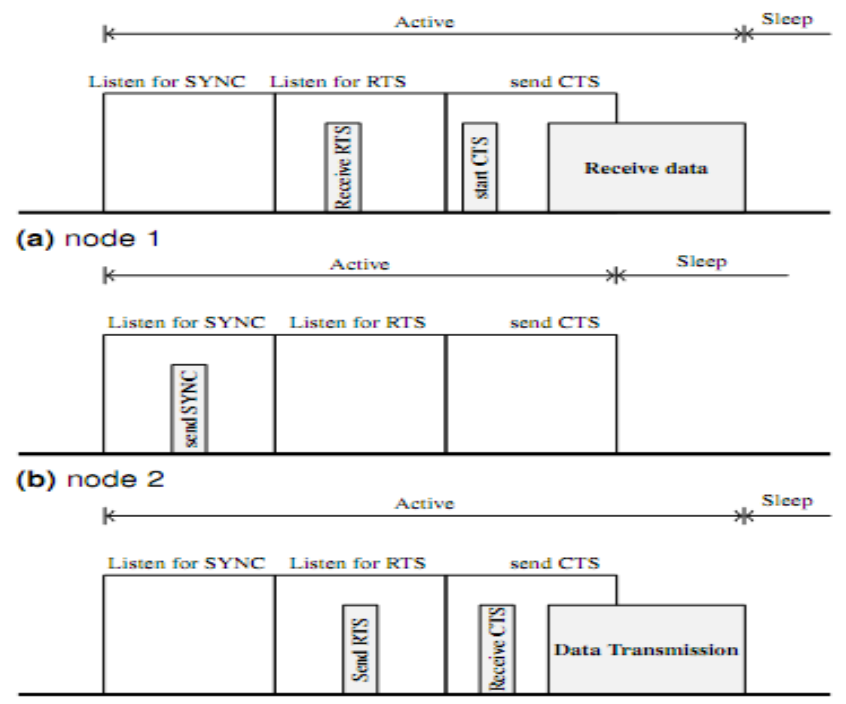

(c) node 3

Figure 5. S-MAC Protocol

\section{HYBRID MAC PROTOCOL (HMAC)}

To overcome the short comings of Carrier Sense Multiple Access (CSMA) and Time division Multiple Access (TDMA), HMAC improves channel utilization and latency under different network conditions such as node density, scalability and traffic in the network. HMAC introduces the concept of slotted frame structure in which the slot for individual frame structures is dynamically shared. A slotted frame structure helps to improve energy efficiency. In HMAC, slotted frame structure needs time synchronization with in each node two hop neighbour. HMAC also supports one hop broadcast mechanism. HMAC Frame Structure is given in Figure 6.

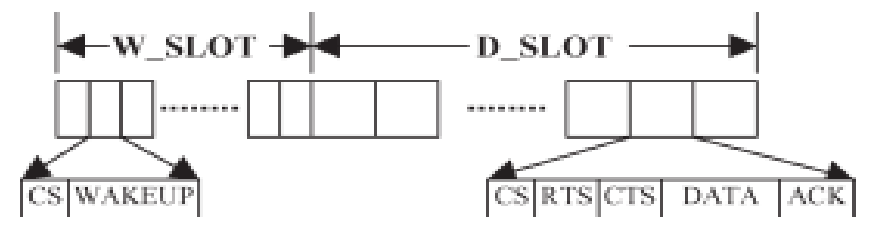

Figure 6. HMAC Frame Structure

HMAC is similar to SMAC with only one difference of slotted sleep time

In HMAC, time frame $T_{x}$ is given by

$$
\mathrm{T}_{\mathrm{x}}=\mathrm{t}_{\text {active }}+\mathrm{Ct}_{\mathrm{s}, \mathrm{n}}
$$

Where $\mathrm{C}$ is the number of equal length slot and $\mathrm{t}_{\text {active }}$ is the listen time same as S-MAC. During the $\mathrm{t}_{\text {active }}$ time HMAC can reserve the slots for nr hops within the same $\mathrm{T}$, so the delay for one hop transmission over $\mathrm{n}_{\mathrm{r}}$, hops is given by

$$
\mathrm{D} 1=\mathrm{T} / \mathrm{n}_{\mathrm{r}}
$$

Average latency of HMAC is given by, 
International Journal of Computer Networks \& Communications (IJCNC) Vol.5, No.2, March 2013

$$
\begin{aligned}
\mathrm{E}[\mathrm{D}(\mathrm{N})] & \approx \mathrm{E}\left[\sum_{n=1}^{N} D_{1}\right] \\
& \approx \mathrm{N}\left(\mathrm{T} / \mathrm{n}_{\mathrm{r}}\right)=\left[\mathrm{N}\left(\mathrm{T} / \mathrm{n}_{\mathrm{r}}\right)\right]
\end{aligned}
$$

MAC is an important technique. It tries to improve the energy efficiency also avoid collisions and retransmission. MAC protocol is used to provide the high throughput and QOS .SMAC improves the energy efficiency by dividing the time into large frames. Each frame has an active part and sleep part. Using the two different parts SMAC saves unnecessary waste of energy efficiency. However, HMAC protocol not only reduces the comparable amount of energy but also provides better QOS.

\section{SPATIAL CORRELATION IN WiRELESS SENSOR NETWORKS}

WSN are event based systems that rely on the collective report of several small sensor nodes observing a physical phenomenon. Applications related to WSN require spatially dense sensor deployment to achieve coverage. Several sensor nodes record information about a single event in a sensor field. Due to elevated density in the topology of WSN, the record created by the sensor nodes may be spatially correlated and subject to an event. The degree of spatial correlation increases with decreasing internode separation.

To develop the spatial correlation in WSN can lead to significant performance improvement of communication protocols. Spatially separated sensor nodes is more useful than the highly correlated data from closely located sensor nodes to the sink. A smaller number of sensor data might be adequate to transmit certain event features to the sink with in a distortion constraint

Each sensor node in the sensor field observes the noisy version of a physical phenomenon. The sink is interested in observing the physical phenomenon using the observations from sensor nodes with the highest accuracy. The physical phenomenon is modeled as a spatio-temporal process $(\mathrm{t} ; \mathrm{x}$ ; $y)$ as a function of time $t$ and spatial coordinates $(x ; y)$.

Depending on the special application, the physical phenomenon may be a spatio-temporal process generated by a point source. The sink is reconstructing the source signal at a specific location based on sensor observations. Although the reconstruction of signal is application specific, the properties of the observations can be modeled based on the spatio temporal process.

Generally the covariance model can be classified into four types .they are

1. Spherical Model

2. Power Exponential Model

3. Rational Quadratic Model

4. Matern Model

Proposed work considers Power exponential model and the covariance function is given by the equation (12),

$$
K_{v}^{P E}(d)=e^{\left(-d / \theta_{1}\right)^{\theta_{2}}} ; \theta_{1}>0, \theta_{2} \in(0,2]
$$

In Equation (12), $\mathrm{K}_{\mathrm{v}}$ represents covariance function and PE represents power exponential model. For $\theta_{2}=1$, the model becomes exponential, while for $\theta_{2}=2$ the model becomes squared 
exponential. The parameter $\theta_{1}$ is important because it controls the relationship between the distance $\mathrm{d}_{\mathrm{i}, \mathrm{j}}$ and $\rho_{\mathrm{i}, \mathrm{j}}$.

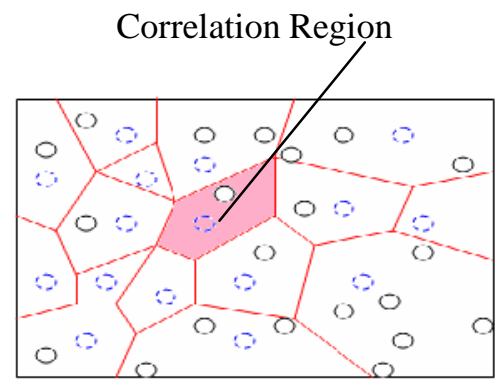

Figure.7 Representation of Correlation Region

Figure.7 shows the representation of correlation region.

$$
\operatorname{corr}\left\{\mathrm{S}_{\mathrm{i},}, \mathrm{S}_{\mathrm{j}}\right\}=\rho_{\mathrm{i}, \mathrm{j}}=\mathrm{K}_{\mathrm{v}}\left(\mathrm{d}_{\mathrm{i}, \mathrm{j}}\right)=\frac{\mathrm{E}\left[\mathrm{S}_{\left.\mathrm{i}, \mathrm{S}_{\mathrm{i}}\right]}\right.}{\sigma^{2} \mathrm{~s}_{\mathrm{s}}}
$$

Equation (13) gives the correlation between the two nodes $n_{i}$ and $n_{j}$ located at the coordinates $S_{i}$, $S_{j}$. where $d_{i, j}=\rho_{s i}-\rho_{s j}$. where $\rho$ denotes the distance between nodes $n_{i}$ and $n_{j}$ respectively, and $k_{v}(\cdot)$ is the correlation model or covariance model. The covariance function is assumed to be non-negative and decreases monotonically with the distance.

Spatial Correlation between the sensor nodes helps to prevent redundant data during transmission. In wireless sensor network, when an event occurs in a sensor field, the nodes which are very nearer to that event area detect the event information and it is sensed by the neighborhood nodes. Every node transmits its own data to sink which is highly correlated that results in redundant transmission. It is not requisite for all the nodes in the sensor field to send the data to the sink. Redundant transmission in a network is reduced by decreasing the selecting the subset of sensor nodes. The subset nodes efficiently transmit the data to sink. The filtration of redundant data and the maximization of network lifetime are increased by designing the efficient MAC protocol. The spatial correlation region is defined as the region in which all the sensor nodes send the readings which are similar in nature and therefore it is enough to send a single report to represent the correlation region.

Total number of nodes available in an event area is N, where the event source is S. Each node in the sensor field observes the noisy version of event information $\mathrm{X}_{\mathrm{i}}(\mathrm{n}), \mathrm{S}_{\mathrm{i}}(\mathrm{n})$ is spatially correlated to event source $\mathrm{S}$. Each node has to encode its observation for the purpose of reporting an event information to sink node. Sink is available at the other end which is responsible for decoding the information to get an estimated value of $\hat{S}$. At a time $\mathrm{n}$, each observed sample is denoted as $\mathrm{X}_{\mathrm{i}}(\mathrm{n})$, equation is formed as,

$$
X_{i}[n]=S_{i}[n]+N_{i}[n]
$$

where the symbol i denotes the spatial location of the node $n_{i}$, i.e. $\left(x_{i}, y_{i}\right), S_{i}(n)$ is the realization of the space-time process $s(t, x, y)$ at time $t=t_{n}$ and $(x, y)=\left(x_{i}, y_{i}\right)$ and $N_{i}(n)$ is the observation noise [1] . $\left\{\mathrm{N}_{\mathrm{i}}(\mathrm{n})\right\}$ is a sequence of i.i.d Gaussian random variables of zero mean and variance $\sigma_{N}^{2}$. We 
assume that the noise each sensor node encounters is independent of each other, i.e., $\mathrm{N}_{\mathrm{i}}(\mathrm{n})$ and $\mathrm{N}_{\mathrm{j}}(\mathrm{n})$ are independent .Each observation $\mathrm{Xi}(\mathrm{n})$ is then encoded into $\mathrm{Yi}(\mathrm{n})$ by the source-coding at the sensor node as,

$$
Y_{i}[n]=f_{i}\left(X_{i}[n]\right)
$$

The information is transferred to the sink through the network. The sink on the other hand decodes the received data to reconstruct an estimation $\hat{S}$ of the source $\mathrm{S}$.

$$
\hat{S}=g\left(Y_{1}\left[n_{1}\right], \ldots, Y_{1}\left[n_{\tau}\right] ; \ldots ; Y_{N}\left[n_{1}\right], \ldots, Y_{N}\left[n_{\tau}\right]\right)
$$

Based on the data received from $\mathrm{N}$ nodes in the event area over a time period $\tau$, the time difference between $t_{n 1}$ and $t_{n \tau}$ is expressed as,

$$
\tau=t_{n_{\tau}}-t_{n_{1}}
$$

The selection of locations of correlated points based on a distortion constraint has been analyzed by means of Vector Quantization (VQ) algorithm. Code book and partition are initially identified by VQ algorithm. Based on the values of code book and partition the distortion value is reduced . The code book represents the locations of the representative node and partition represents the areas of which representative nodes are responsible. Significant improvement is achieved in distortion by selecting the location of representative node using VQ algorithm. The spatial correlation region is defined as the region in which all the sensor nodes send the readings which are similar in nature and therefore it is enough to send a single report to represent the correlation region. Correlation Radius and Correlation Region are two important observations in spatial correlation based iterative node selection algorithm.

- Correlation Radius - It is represented by $\mathrm{r}_{\text {corr }}$. It is the radius of correlation region

- Correlation Neighbor - A node $\mathrm{n}_{\mathrm{j}}$ is said to be the correlation neighbor of node $n_{i}$ if the distance $d_{i, j}$ of the node $n_{i}$ is smaller than $\mathrm{r}_{\text {corr }}$

Spatial correlation based S-MAC is compared with spatial correlation based H-MAC in the proposed work. The parameters considered for the analysis are end to end delay, Packet delivery ratio, Throughput, Energy consumption. The performance analysis is given in Section 7.

\section{RESULTS AND DISCUSSIONS}

An Energy Efficient Analysis of S-MAC And HMAC Protocols For Wireless Sensor Networks is proposed and implemented using the simulation tool NS2.The Parameters such as End to End delay, Packet delivery ratio, Throughput and Energy Consumption have been compared with SMAC and H-MAC protocols. The network size is considered as $1500 \times 1500$ and totally eight number of hops are involved. $2 \mathrm{MHz}$ of bandwidth and 1000 joules of initial energy with $1 \mathrm{Mbps}$ range of data rate and $1 \mathrm{~mW}$ of transmitting and receiving and Idle power values with $0.01 \mathrm{mw}$ of sleep power are designed to form a network model. In the following sections, the comparison of End to End delay, Packet delivery ratio, Throughput, Energy Consumption with S-MAC and HMAC protocol is discussed briefly. 
International Journal of Computer Networks \& Communications (IJCNC) Vol.5, No.2, March 2013

\subsection{Comparison of End to End delay with S-MAC and HMAC}

End to End delay is defined as the ratio between a sum of individual data packet delay to the total number of data packets delivered.

End to End delay $=[($ Sum of Individual data packet delay $) /($ Total number of data Packets delivered)]

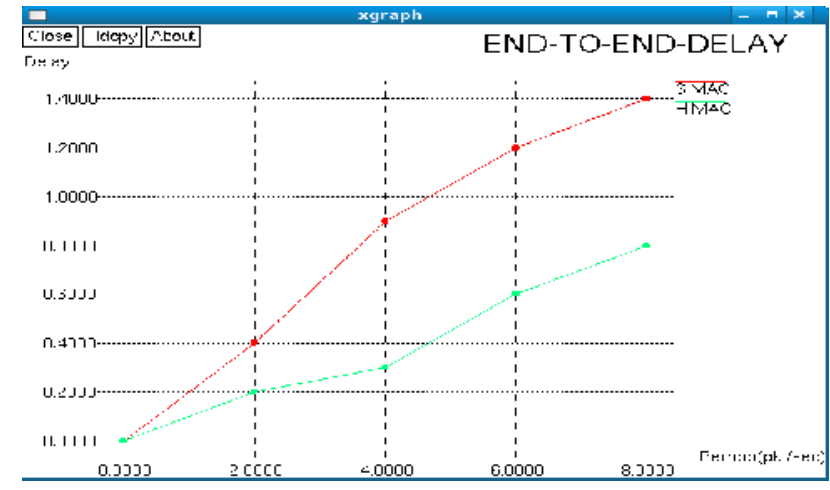

Figure 8. End to End delay Vs Per hop

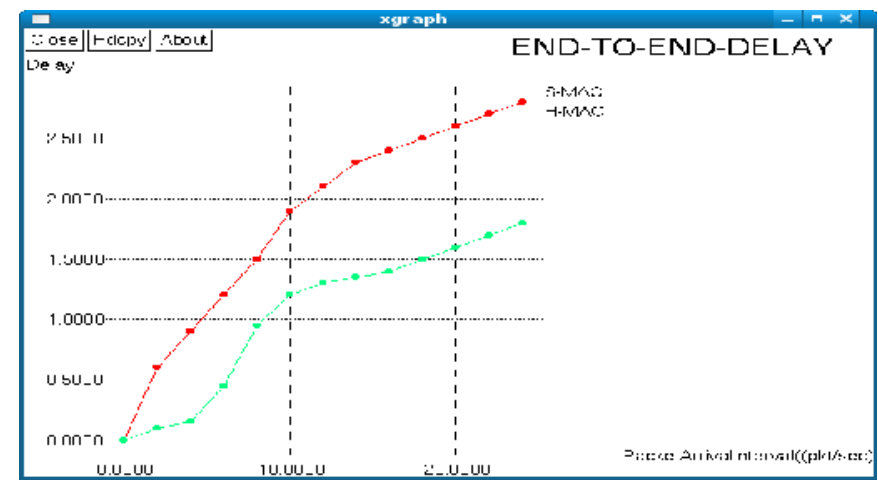

Figure 9. End to End delay Vs Packet Arrival interval

\subsection{Comparison of Packet Delivery Ratio with S-MAC and HMAC}

Packet Delivery ratio is the percentage of the ratio between total number of data packets successfully delivered to the total number of data packets sent.

$\mathrm{Pdr}=[($ Total number of data packets successfully delivered) $/$ (Total number of data packets sent ) ) $100 \%$ 
International Journal of Computer Networks \& Communications (IJCNC) Vol.5, No.2, March 2013

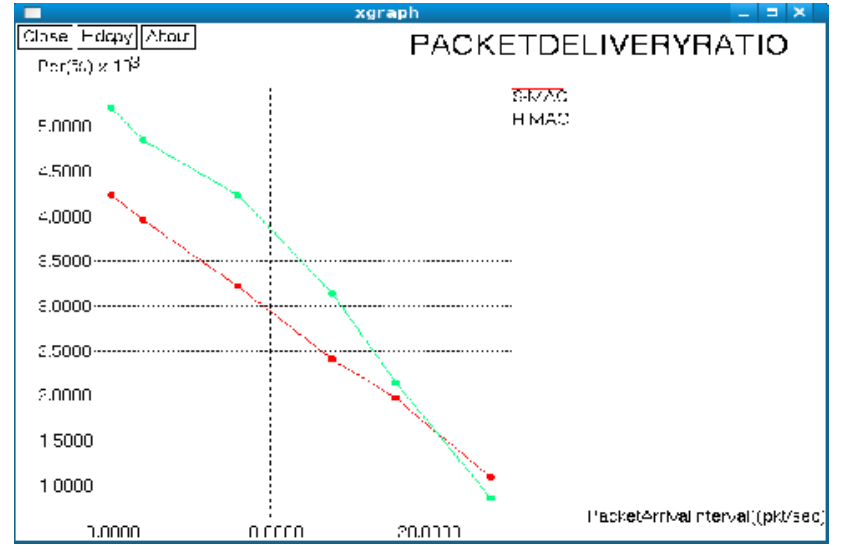

Figure 10. Packet Delivery Ratio

\subsection{Comparison of Throughput with S-MAC and H-MAC}

Throughput is defined as the number of packets delivered at sink node per time unit

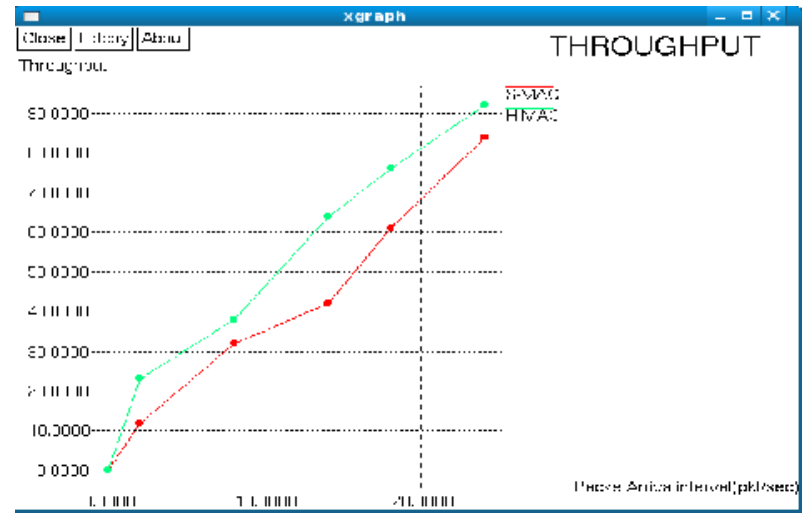

Figure 11. Throughput

\subsection{Comparison of Energy Consumption with S-MAC and H-MAC}

Energy Consumption is defined as the ratio between sum of energy expended by each node to the total number of data packets delivered.

Energy Consumption $=[($ Sum of Energy expended by each node $) /($ Total number of data packets delivered)] 
International Journal of Computer Networks \& Communications (IJCNC) Vol.5, No.2, March 2013

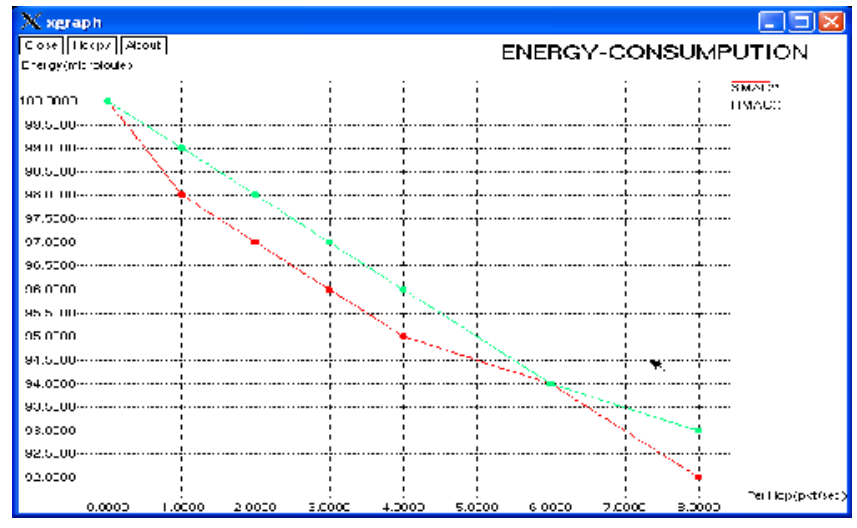

Figure 12. Energy Consumption

As shown Figure 8\&9, the End to End delay value for S-MAC will be higher than compared with the H-MAC for each packet arrival interval and for each hop. As shown in Figure 10, the Packet delivery ratio for H-MAC is very high, at $20 \mathrm{PAI}$, the Packet delivery ratio is $12 \%$ higher than that of S-MAC. As shown in Figure 11, the H-MAC protocol gives 8\% higher value of Energy Consumption \& 9\% higher value of throughput than S-MAC protocol. As shown in Figure 12, HMAC protocol gives 50\% higher energy consumption than that of S-MAC. As shown in Figures $8,9,10,11,12$ the performance of H-MAC is higher than that of S-MAC protocol because H-MAC has the best time synchronization and full duplex communication. It has the ability to change according to the environment. Therefore it is suitable for broadcast and convergecast.

\section{CONCLUSION AND FUTURE WORK}

An Energy Efficient Analysis of S-MAC and H-MAC Protocols for Wireless Sensor Networks is proposed in this paper. The concept of S-MAC and the concept of H-MAC protocols are analyzed individually using spatial correlation with the simulation tool NS2.The parameters considered for this analysis are End to End delay, Packet delivery ratio, Throughput and Energy Consumption. For various set of values the performance of S-MAC and H-MAC is analyzed. The results show that H-MAC protocol performance is better than that of S-MAC protocol. In future, different types of MAC protocol performance will be analyzed and the values of energy consumption for each node will be tabulated.

\section{REFERENCES}

[1] Lanny Sitanayah,Cormac J.Sreenan,Kenneth N.Brown, “A Hybrid MAC Protocol for Emergency Response Wireless Sensor Networks”, SENSORCOMM 2010, 2010,pp-244- 249.

[2] Lamia Chaari, Lotif "Wireless Sensors Networks MAC protocols analysis" Journal of Telecommunications, Vol 2, No 1,April 2010.

[3] Somnath Ghosh,Prakasj Veeraraghavan,Samar Singh,Lei Zhang "Performance of a Wireless sensor Network MAC Protocol with a Global Sleep Schedule” International journal of Multimedia and Ubiquities Engineering, Vol 4,No 2,April 2009.

[4] Nidhi Batra,Anuj j jain,susurender Dhiman "An Optimized Energy Efficient Routing Algorithm for WSN” Innovative Journal of Innovative Technology and Creative Engg,Vol.1,No.5 May 2011.

[5] Ilker Demirkol, Cem Ersoy and Fathih ala,"MAC Protocols for Wireless Sensor Networks: A survey”,IEEE Communication Magazine,Vol 44,No.4,2006,pp.115121.http:dx.doi.org/10.1109/MCOM.2006.1632658. 
International Journal of Computer Networks \& Communications (IJCNC) Vol.5, No.2, March 2013

[6] Uros Pesovic, Aleksandar Peulic,Zarko Cucej "MAC Protocols for Wireless Sensor Network",Electro Technical Review,2008,pp. 50-55.

[7] Heping Wang, Xiaobo Zhang,Farid Nait-Abdesesselam, "Cross Layer Optimized MAC to support Multihop QOS routing for Wireless Sensor Network", IEEE Transactions on Vehicular Technology, 2010,vol. 59, No.5, http://dx.doi.org/10.1109/TVT.2010.2042185.

[8] Ines Slama,Badii Jouber,Djamal Zeghlache "Priority based Hybrid MAC for Energy Efficiency in Wireless Sensor Networks",Wireless Sensor Networks,2010,Vol.2,pp-755-767.

[9] M.Miladi,T.Ezzedine,R.Bouallegue, "Hybrid MAC Protocols Characteristics in Multi-hops Wireless Sensor Networks", International journal of Electrical and Computer Engineering,Vol.3,No.3, 2008.

[10] Chalmtac A.farag, A.D.Myers, V.R.Syrotiuk,G.Zkruba, "A Performance Comparison of Hybrid and Conventional MAC Protocols for Wireless Networks", Vehicular Technology Conference Proceedings VTC 2000, vol.1,pp.201-205. 